\title{
Natural Inhibitors of the Aromatase Enzyme*
}

\section{Roberto R. Gil}

Departamento de Química Orgánica e IMBIV-CONICET, Facultad de Ciencias Químicas, Universidad Nacional de Córdoba. Ciudad Universitaria, 5000 Córdoba, Argentina

E-mail: gilrr@dqo.fcq.unc.edu.ar

Abstract: The results of three years of search for natural aromatase inhibitors will be presented.

Estrogen biosynthesis is catalyzed by the aromatase enzyme complex. This complex if made up of a member of the cytochrome P450 superfamily of enzymes known as cytochrome P450 aromatase (P450arom, produced by the CYP19 gene). Associated with this enzyme one finds the flavoprotein NADPH cytochrome P450 reductase. These two enzymes catalyze the aromatization of the A ring of androgens to form the characteristic phenolic ring of the estrogens. This reaction involves three sequential oxidations of the C-19 methyl group of the substrate (testosterone and androstenedione), its elimination as formic acid and the aromatization of the A ring to afford $17 \beta$-estradiol y estrone, respectively. This is the only reaction found in vertebrates in which an aromatic ring is introduced in a molecule. The regulation of this enzyme plays an important role in several physiological processes and in certain diseases, the most important of which is hormone dependent breast cancer. The use of aromatase inhibitors to treat this disease is now very commonplace. There are two kinds of inhibitors, competitive ones and suicide inhibitors; both types include both steroidal and non-steroidal compounds. Due to their great therapeutic importance, presently the list of inhibitors is quite long, but only five have been shown to be relatively safe and to possess reasonable clinical efficacy. These are: aminoglutetimide, 4-hydroxyandrostenedione, anastrozol, letrozol y vorozol; the last three being third generation non-steroidal inhibitors. On the other hand, a few plant secondary metabolites have shown significant inhibitory activity towards the aromatase enzyme. For this reason we consider the search for and characterization of novel natural products with this type of activity to be interesting.

At this conference we will present the results of three years of joint research with colleagues from the Department of Clinical Biochemistry of our faculty, which may be summarized as follows:

a) A group of sesquiterpene lactones isolated from different species of Asteraceae inhibited the activity of the aromatase enzyme in human placenta microsomes. The three most active compounds were the guaianolides 10-epi-8-desoxycumambrine B, dehydroleucodine and ludartine.

b) Complete kinetic studies of these compounds as well as differential UV-Vis studies, were done to measure their binding affinity towards the iron of the heme group found in the active site of the enzyme. 
c) To measure the specificity of each inhibitor, their activity towards two other enzymes of the steroidigenic cascade was evaluated.

d) Reduction of the characteristic exocyclic double bond of the most active compound, 10-epi-8desoxycumambrine $\mathrm{B}$, allowed us to prepare a dihydro derivative that retained the capability to inhibit the aromatase enzyme while at the same time displaying none of the precursor's cytotoxicty. This allowed us to successfully evaluate the inhibition of the enzyme found in JEG-3 choriocarcinome cells. It is important to mention that the literature to date contains reports of nearly 4,000 sesquiterpene lactones. Many of them display several types of biological activity, but always associated with the presence of the characteristic exocyclic double bond. In our case this is not so, which represents an important pharmacological novelty.

e) The results, combined with molecular modeling studies, allow us to propose an inhibition mechanism that shows that the exocylic double bond does not participate.

f) Semisynthetic derivatives were prepared from ludartine with the purpose of speculating about structure-activity relationships.

*Note: Translation by the Editorial Staff. 